\title{
THE RELATION BETWEEN CIRCULAR VELOCITY AND CENTRAL VELOCITY DISPERSION IN LOW SURFACE BRIGHTNESS GALAXIES
}

\author{
E.M. Corsini, A. Pizzella, E. Dalla Bonta', F. Bertola \\ Dipartimento di Astronomia, Universita ' di Padova, Italy \\ corsini@pd.astro.it \\ L. Coccato \\ Kapteyn Astronomical Institute, Groningen, The Netherlands \\ M. Sarzi \\ Physics Department, University of Oxford, UK
}

\begin{abstract}
We analyzed a sample of high and low surface brightness (HSB and LSB) disc galaxies and elliptical galaxies to investigate the correlation between the circular velocity $\left(V_{c}\right)$ and the central velocity dispersion $\left(\sigma_{c}\right)$. We better defined the previous $V_{c}-\sigma_{c}$ correlation for HSB and elliptical galaxies, especially at the lower end of the $\sigma_{c}$ values.

Elliptical galaxies with $V_{c}$ based on dynamical models or directly derived from the $\mathrm{H}$ I rotation curves follow the same relation as the HSB galaxies in the $V_{c}-\sigma_{c}$ plane. On the contrary, the LSB galaxies follow a different relation, since most of them show either higher $V_{c}$ (or lower $\sigma_{c}$ ) with respect to the HSB galaxies. This argues against the relevance of baryon collapse in the radial density profile of the dark matter haloes of LSB galaxies. Moreover, if the $V_{c}-\sigma_{c}$ relation is equivalent to one between the mass of the dark matter halo and that of the supermassive black hole, these results suggest that the LSB galaxies host a supermassive black hole with a smaller mass compared to HSB galaxies of equal dark matter halo. On the other hand, if the fundamental correlation of SMBH mass is with the halo $V_{c}$, then LSBs should have larger black hole masses for given bulge $\sigma_{c}$.
\end{abstract}

Keywords: galaxies: elliptical and lenticular, $\mathrm{cD}$ - galaxies: fundamental parameters galaxies: kinematics and dynamics - galaxies: spirals 


\section{Introduction}

A possible relation between the central velocity dispersion of the spheroidal component $\left(\sigma_{c}\right)$ and the galaxy circular velocity $\left(V_{c}\right)$ measured in the flat region of the rotation curve (RC) was suggested by Whitmore et al. (1979). By measuring stellar velocity dispersions and $\mathrm{H}$ I line widths for a sample of 19 spiral galaxies they found a a significant decrease in $V_{c} / \sigma_{c}$ with increasing bulge-to-disk ratio. Since $\sigma_{c}$ and $V_{c}$ probe the potential of the spheroid and dark matter (DM) halo, a mean value $V_{c} / \sigma_{c} \simeq 1.7$ implies these components are dynamically separate with the bulge substantially cooler than halo.

Gerhard et al. (2001) derived the $V_{c^{-}} \sigma_{c}$ relation for the sample of giant ellipticals studied by Kronawitter et al. (2000). This was explained as an indication of near dynamical homology of these objects which were selected to be nearly round and almost non-rotating elliptical galaxies. These galaxies form a unique dynamical family which scales with luminosity and effective radius. As a consequence the maximum $V_{c}$ of the galaxy is correlated to its $\sigma_{c}$. Whether the same is true for more flattened and fainter ellipticals is still to be investigated. On the contrary, both shape and amplitude of the RC of a spiral galaxy depend on the galaxy luminosity and morphological type (e.g., Burstein $\&$ Rubin 1985). As a consequence for spiral galaxies the $V_{c}-\sigma_{c}$ relation is not expected a priori.

Nevertheless, Ferrarese (2002) and Baes (2003) found that elliptical and spiral galaxies define a common $V_{c}-\sigma_{c}$ relation. In particular, it results that for a given $\sigma_{c}$ the value of $V_{c}$ is independent of the morphological type. But $\sigma_{c}$ and $V_{c}$ are related to the mass of the supermassive black hole (hereafter SMBH, see Ferrarese \& Ford 2005 for a review) and DM halo (e.g., Seljak 2002), respectively. Therefore Ferrarese (2002) argued that the $V_{c}-\sigma_{c}$ relation is suggestive of a correlation between the mass of SMBH and DM halo.

Previous works concentrated on high surface brightness (HSB) galaxies. It is interesting to investigate whether the $V_{c}-\sigma_{c}$ relation holds also for less dense objects characterized by a less steep potential well. This is the case of low surface brightness (LSB) galaxies, which are disc galaxies with a central faceon surface brightness $\mu_{B} \geq 22.6 \mathrm{mag} \operatorname{arcsec}^{-2}$ (e.g., Impey et al. 1996). In Pizzella et al. (2005) we studied the behavior of LSB galaxies in the $V_{c^{-}}-\sigma_{c}$ relation. Here we present our results.

\section{Sample selection}

In the past years we started a scientific program aimed at deriving the detailed kinematics of ionized gas and stars in HSB and LSB galaxies in order to study their mass distribution and structural properties (e.g., Pignatelli et al. 2001). 
We measured the velocity curves and velocity dispersion profiles along the major axis for both the ionized-gas and stellar components for a preliminary sample of 50 HSB galaxies [10 S0-S0/a's in Corsini et al. (2003); 7 Sa's in Bertola et al. (1996) and Corsini et al. (1999); 16 S0-Sc's in Vega Beltran et al. (2001); 17 Sb-Scd's in Pizzella et al. (2004a)] and 11 LSB galaxies (Pizzella et al. 2004b). The HSB sample consists of disc galaxies with Hubble type ranging from S0 to Scd, an inclination $i \geq 30^{\circ}$ and a distance $D<80 \mathrm{Mpc}$. The LSB sample consists of disc galaxies with Hubble type ranging from Sa to Irr, an intermediate inclination $\left(30^{\circ} \leq i \leq 70^{\circ}\right)$, and a distance $D<65 \mathrm{Mpc}$. In order to complete our sample of disc galaxies we included the $38 \mathrm{Sa}-\mathrm{Scd}$ galaxies previously studied by Ferrarese (2002) and the $12 \mathrm{Sb}-\mathrm{Sc}$ galaxies by Baes et al. (2003).

Finally, we considered a sample of 24 elliptical galaxies with a flat RC and for which both $V_{c}$ and $\sigma_{c}$ are available from the literature. They include 19 objects studied by Kronawitter et al. (2000) who derived $V_{c}$ by dynamical modeling and 5 objects studied by Bertola et al. (1993) for which $V_{c}$ is directly derived from the flat portion of their H I RCs. The addition of these galaxies will allow to test against model-dependent biases in the $V_{c}-\sigma_{c}$ relation.

\section{Measuring $V_{c}$ and $\sigma_{c}$}

For all the disc galaxies we obtained the ionized-gas RC. We rejected galaxies with asymmetric RCs or RCs which were not characterized by an outer flat portion. The flatness of each RC has been checked by fitting it with a linear law $V(R)=A R+B$ for $R \geq 0.35 R_{25}$. The radial range has been chosen in order to avoid the bulge-dominated region. The RCs with $|A| \geq 2$ $\mathrm{km} \mathrm{s}^{-1} \mathrm{kpc}^{-1}$ within $3 \sigma$ have been considered not to be flat. We derived $V_{c}$ by averaging the outermost values of the flat portion of the RC. We derived $\sigma_{c}$ from the stellar kinematics by extrapolating the velocity dispersion radial profile to the centre. This has been done by fitting the innermost data points with an empirical function (either an exponential, or a Gaussian or a constant). We did not apply any aperture correction to $\sigma_{c}$ as discussed by Pizzella et al. (2004a).

For the elliptical galaxies we obtained $V_{c}$ either from the dynamical models by Gerhard et al. (2001) or from the flat portion of the H I RC. For these galaxies we relaxed the flatness criterion in favor of their large radial extension which is about 10 times larger than that of optical RCs. Aperture measurements of the stellar velocity dispersion corrected to $r_{e} / 8$ were adopted to estimate $\sigma_{c}$.

\section{The $V_{c}-\sigma_{c}$ relation for HSB and elliptical galaxies}

In summary, we have 40 disc galaxies [ 15 from our preliminary sample, 16 from Ferrarese (2002), and 9 from Baes et al. (2003) with flat RCs extending 
to $\sim 0.8 R_{25}$ ] and 24 elliptical galaxies [19 from Kronawitter et al. (2000) with flat RCs extending to about $\sim 0.5 R_{25}, 5$ from Bertola et al. (1993) with flat H I RCs extending to $\sim 3 R_{25}$ ].

A power law has been usually adopted to describe the correlation between $V_{c}$ and $\sigma_{c}$ for galaxies with $\sigma_{c}>80 \mathrm{~km} \mathrm{~s}^{-1}$. We find

$$
\log V_{c}=(0.74 \pm 0.07) \log \sigma_{c}+(0.80 \pm 0.15)
$$

with $V_{c}$ and $\sigma_{c}$ expressed in $\mathrm{km} \mathrm{s}^{-1}$. The resulting power law is plotted in Fig. 1 (dash-dotted line). However, according to a $\chi^{2}$ analysis the data are also consistent with a linear law out to velocity dispersions as low as $\sigma_{c} \approx 50$ $\mathrm{km} \mathrm{s}^{-1}$

$$
V_{c}=(1.32 \pm 0.09) \sigma_{c}+(46 \pm 14) .
$$

The resulting straight line is plotted in Fig. 1 (continuous line).

The data points corresponding to the 5 elliptical galaxies with $V_{c}$ based on $\mathrm{H}$ I data follow the same relation as the remaining disc and elliptical galaxies. They are mostly located on the upper end of the $V_{c}-\sigma_{c}$ relation derived for disc galaxies, in agreement with the findings of Bertola et al. (1993). They studied these elliptical galaxies and showed that their DM content and distribution are similar to those of spiral galaxies.

\section{The $V_{c}-\sigma_{c}$ relation for $L S B$ galaxies}

The LSB and HSB galaxies do not follow the same $V_{c}-\sigma_{c}$ relation. In fact, most of the LSB galaxies are characterized by a higher $V_{c}$ for a given $\sigma_{c}$ (or a lower $\sigma_{c}$ for a given $V_{c}$ ) with respect to HSB galaxies. By applying to the LSB data points the same regression analysis which has been adopted for the HSB and elliptical galaxies of the final sample, we find

$$
V_{c}=(1.35 \pm 0.19) \sigma_{c}+(81 \pm 23)
$$

with $V_{c}$ and $\sigma_{c}$ expressed in $\mathrm{km} \mathrm{s}^{-1}$. The straight line corresponding to this fit, which is different from the one obtained for HSB and elliptical galaxies and happens to be parallel to it, is plotted in Fig. 1 (dotted line). To address the significance of this result, which is based only on 8 data points, we compared the distribution of the normalized scatter of the LSB galaxies to that of the HSB and elliptical galaxies. They are different at a high confidence level $(>99 \%)$ by a Kolmogorov-Smirnov test. The fact that these objects fall in a different region of the $V_{c}-\sigma_{c}$ plane confirms that LSB and HSB galaxies constitute two different classes of galaxies.

\section{Conclusions}

Both demographics of SMBHs and study of DM distribution in galactic nuclei benefit from the $V_{c}-\sigma_{c}$ relation. The recent finding that the mass of 


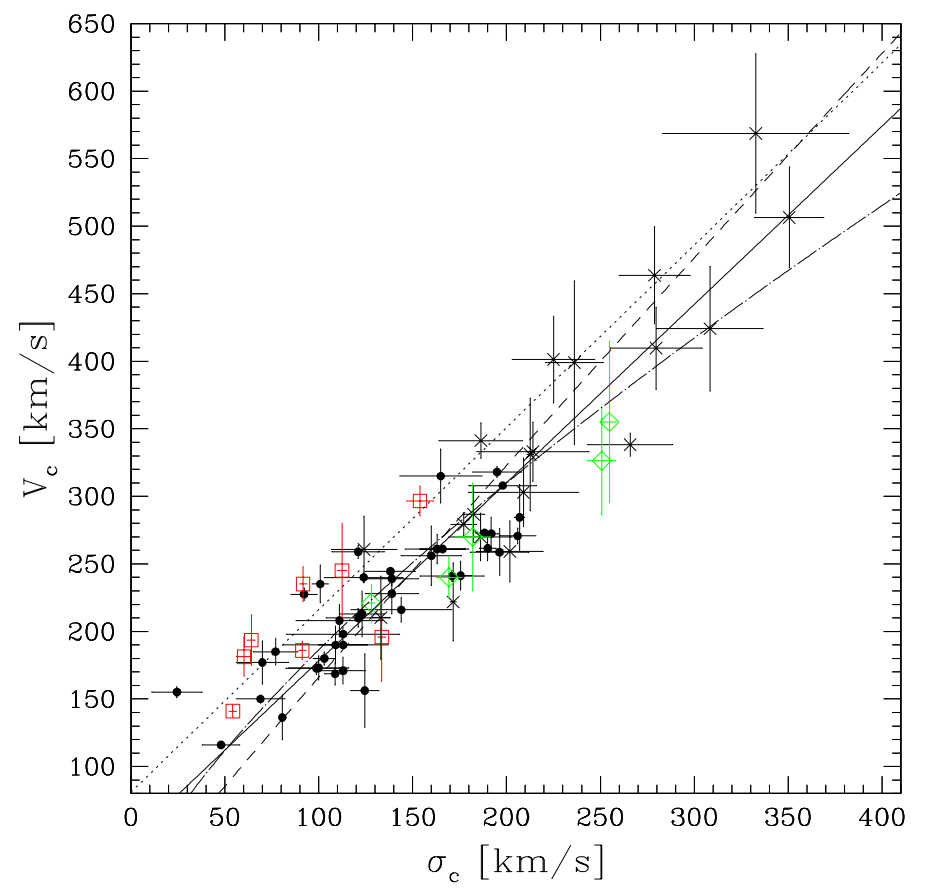

Figure 1. The correlation between $V_{c}$ and $\sigma_{c}$ The data points corresponding to HSB galaxies (filled circles), LSB galaxies (squares), and elliptical galaxies with $V_{c}$ obtained from $\mathrm{H}$ I data (diamonds) or dynamical models (crosses) are shown. The dash-dotted and continuous line represent the power-law (Eq. 1) and linear fit (Eq. 2) to HSB and elliptical galaxies. The dotted line represents the linear-law fit (Eq. 3) to LSB galaxies. For a comparison, the dashed line corresponds to the power-law fit to spiral galaxies with $\sigma_{c}>80 \mathrm{~km} \mathrm{~s}^{-1}$ by Baes et al. (2003).

SMBHs correlates with different properties of the host spheroid supports the idea that formation and accretion of SMBHs are closely linked to the formation and evolution of their host galaxy. A task to be pursued is to obtain a firm description of all these relationships spanning a wide range of SMBH masses and address if they hold for all Hubble types. In fact, the current demography of SMBHs suffers of important biases, related to the limited sampling over the different basic properties of their host galaxies. The finding that the $V_{c}-\sigma_{c}$ relation holds for small values of $\sigma_{c}$ points to the idea that SMBHs with masses $\mathrm{M}_{\bullet}<10^{6} \mathrm{M}_{\odot}$ may also exist and follow the $\mathrm{M}_{\bullet}-\sigma$ relation.

Moreover, it has been suggested that the $V_{c}-\sigma_{c}$ relation is equivalent to one between the masses of SMBH and DM halo (Ferrarese 2002; Baes 2003) because $\sigma_{c}$ and $V_{c}$ are related to the masses of the central SMBH and DM halo, respectively. Yet, this claim is to be considered with caution, as the 
demography of SMBHs is still limited, in particular as far as spiral galaxies are concerned. Furthermore, the calculation of the virial mass of the DM halo from the measured $V_{c}$ depends on the assumptions made for the DM density profile and the resulting rotation curve (e.g., see the prescriptions by Seljack et al. 2002). A better estimate of the virial velocity of the DM halo $V_{\text {vir }}$ can be obtained by constraining the baryonic-to-dark matter fraction with detailed dynamical modeling of the sample galaxies. The resulting $V_{v i r}-\sigma_{c}$ relation is expected to have a smaller scatter than the $V_{c}-\sigma_{c}$ relation. If the $\mathrm{M}_{\bullet}-\sigma$ relation is to hold, the deviation of LSB galaxies with bulge from the $V_{c}-\sigma_{c}$ of HSB and elliptical galaxies suggests that for a given DM halo mass the LSB galaxies would host a SMBH with a smaller mass compared to HSB galaxies. On the other hand, if the fundamental correlation of SMBH mass is with the halo $V_{c}$, then LSBs should have larger black-hole masses for given bulge $\sigma_{c}$. This should be accounted for in the theoretical and numerical investigations of the processes leading to the formation of LSB galaxies.

The collapse of baryonic matter can induce a further concentration in the DM distribution, and a deepening of the overall gravitational well in the central regions. If this is the case, the finding that at a given DM mass (as traced by $V_{c}$ ) the central $\sigma_{c}$ of LSB galaxies is smaller than in their HSB counterparts, would argue against the relevance of baryon collapse in the radial density profile of DM in LSB galaxies.

\section{References}

Baes, M., Buyle, P., Hau, G. K. T., \& Dejonghe, H. 2003, MNRAS, 341, L44

Bertola, F., Cinzano, P., Corsini, E. M., et al. 1996, ApJ, 458, L67

Bertola, F., Pizzella, A., Persic, M., \& Salucci, P. 1993, ApJ, 416, L45

Burstein, D., \& Rubin, V. C. 1985, ApJ, 297, 423

Corsini, E. M., Pizzella, A., Sarzi, M., et al. 1999, A\&A, 342, 671

Corsini, E. M., Pizzella, A., Coccato, L., \& Bertola, F. 2003, A\&A, 408, 873

Ferrarese, L. 2002, ApJ, 578, 90

Ferrarese, L., \& Ford, H. 2005, Space Science Rev., 116, 523

Gerhard, O., Kronawitter, A., Saglia, R. P., \& Bender, R. 2001, AJ, 121, 1936

Kronawitter, A., Saglia, R. P., Gerhard, O., \& Bender, R. 2000, A\&AS, 144, 53

Impey, C. D., Sprayberry, D., Irwin, M. J., \& Bothun, G. D. 1996, ApJS, 105, 209

Pignatelli, E., Corsini, E. M, Vega Beltran, J. C., et al. 2001, MNRAS, 323, 188

Pizzella, A., Corsini, E. M., Vega Beltran, J. C., \& Bertola, F. 2004a, A\&A, 424, 447

Pizzella, A., Corsini, E. M., Bertola, F., et al. 2004b, IAU Symp., 222, 337

Pizzella, A., Corsini, E. M., Dalla Bonta', et al. 2005, ApJ, 631, 785

Seljak, U. 2002, MNRAS, 334, 797

Vega Beltran, J. C., Pizzella, A., Corsini, E. M., et al. 2001, A\&A, 374, 394

Whitmore, B. C., Schechter, P. L., \& Kirshner, R. P. 1979, ApJ, 234, 68 